\title{
Enhanced Auditory Evoked Activity to Self-Generated Sounds Is Mediated by Primary and Supplementary Motor Cortices
}

\author{
Daniel Reznik, Ori Ossmy, and ๑Roy Mukamel \\ School of Psychological Sciences and Sagol School of Neuroscience Tel-Aviv University, Tel-Aviv 69978, Israel
}

Accumulating evidence demonstrates that responses in auditory cortex to auditory consequences of self-generated actions are modified relative to the responses evoked by identical sounds generated by an external source. Such modifications have been suggested to occur through a corollary discharge sent from the motor system, although the exact neuroanatomical origin is unknown. Furthermore, since tactile input has also been shown to modify responses in auditory cortex, it is not even clear whether the source of such modifications is motor output or somatosensory feedback. We recorded functional magnetic resonance imaging (fMRI) data from healthy human subjects $(n=11)$ while manipulating the rate at which they performed sound-producing actions with their right hand. In addition, we manipulated the amount of tactile feedback to examine the relative roles of motor and somatosensory cortices in modifying evoked activity in auditory cortex (superior temporal gyrus). We found an enhanced fMRI signal in left auditory cortex during perception of self-generated sounds relative to passive listening to identical sounds. Moreover, the signal difference between active and passive conditions in left auditory cortex covaried with the rate of sound-producing actions and was invariant to the amount of tactile feedback. Together with functional connectivity analysis, our results suggest motor output from supplementary motor area and left primary motor cortex as the source of signal modification in auditory cortex during perception of self-generated sounds. Motor signals from these regions could represent a predictive signal of the expected auditory consequences of the performed action.

Key words: action consequences; auditory perception; corollary discharge; efference copy; fMRI

\section{Introduction}

Our senses are exposed to a constant flow of sensory inputs. While some of these inputs originate from external changes in the environment, other inputs are the direct result of self-generated actions. Monitoring the source of such changes in sensory input is crucial for the correct assignment of agency and the coupling of actions with their expected sensory consequences (Jeannerod, 2003). Research over the past several years suggests that indeed perception and its underlying activity in sensory cortex do not depend merely on the physical attributes of the stimulus, but also on whether or not it is the consequence of self-generated actions (Wolpert et al., 1995; Poulet and Hedwig, 2007; Schroeder et al., 2010).

Self-generated vocalizations have been reported to result in decreased neural firing rates in primate auditory cortex a few

\footnotetext{
Received Sept. 4, 2014; revised Nov. 30, 2014; accepted Dec. 8, 2014.

Author contributions: D.R., 0.0., and R.M. designed research; D.R. performed research; D.R., 0.0., and R.M. contributed unpublished reagents/analytic tools; D.R. and R.M. analyzed data; D.R., 0.0., and R.M. wrote the paper.

This study was supported by the I-CORE Program of the Planning and Budgeting Committee, and Israel Science Foundation Grant 51/11, Israel Science Foundation Grants 1771/13 and 2043/13, and Human Frontiers Science Project Career Development Award CDA00078/2011-C to R.M.; Sagol School of Neuroscience and the Israeli Presidential Honorary Scholarship for Neuroscience Research to D.R. and 0.0. We thank A. Shuster and laboratory members for fruitful discussion and comments on this manuscript.

Correspondence should be addressed to Roy Mukamel, School of Psychological Sciences and Sagol School of Neuroscience Tel-Aviv University, Tel-Aviv 69978, Israel. E-mail: rmukamel@tau.ac.il.

DOI:10.1523/JNEUROSCI.3723-14.2015

Copyright $\odot 2015$ the authors $\quad 0270-6474 / 15 / 352173-08 \$ 15.00 / 0$
}

hundred milliseconds before vocalization onset (Eliades and Wang, 2003). In mice, the firing rates of neurons in primary visual cortex are modified by the speed of running even when the mice are in complete darkness (Saleem et al., 2013). Such modifications are believed to be caused by an efference copy or a corollary discharge (Sperry, 1950; von Holst, 1954) that is sent by the motor system to sensory regions in parallel to the actual motor command sent to the relevant effector. Such signals have been shown to facilitate, inhibit, or otherwise modify neural responses to the sensory consequences of self-generated actions (for review, see Crapse and Sommer, 2008).

In humans, such modifications have been demonstrated in various modalities both at the behavioral and physiological levels (for review, see Hughes et al., 2013). The magnitude of such modifications in sensory cortices has been previously reported to be mediated by the amount of motor output (Cui et al., 2014), and its source is believed to lie within the motor system, although its exact origin remains unknown. One study points to the ventral pre-motor cortex as a candidate brain region providing efferent signals to somatosensory cortex (Christensen et al., 2007), while other studies point to the cerebellum (Blakemore et al., 1998; Knolle et al., 2012, 2013). Yet another study suggests the involvement of supplementary motor area (SMA; Haggard and Whitford, 2004).

In the case of auditory cortex, another potential source of signal modification is tactile feedback, which is typically coupled 
with sound-producing actions (e.g., clapping hands). Since there is evidence for evoked response in auditory cortex during passive tactile stimulation applied to the hand (Foxe et al., 2002; Schürmann et al., 2006), the roles of motor output and somatosensory feedback in such signal modifications are unclear.

The aim of the current study was to examine candidate neuroanatomical sources underlying modification of auditory cortex responses evoked by self-generated sounds. To this end, we acquired whole-brain functional magnetic resonance imaging (fMRI) data from healthy subjects, while manipulating the amount of motor output and tactile feedback (using a motion detection glove) during the production of self-generated sounds. Our results point to the SMA and left primary motor cortex (M1; contralateral to the sound-producing hand) as candidate sources for signal modification in auditory cortex during active sound generation. Additionally, tactile feedback does not seem to play a significant role in such modifications.

\section{Materials and Methods}

Subjects. Eleven subjects ( 3 males; mean age, 23.3 years; age range, 20-26 years) naïve to the purpose of the experiment were recruited according to standard safety inclusion/exclusion criteria for fMRI studies. All subjects were right handed, and had normal (or corrected to normal) vision and hearing. The study was approved by the ethics committee of Tel Aviv University and the Tel Aviv Sourasky Medical Center. All subjects provided written informed consent to participate in the study and were compensated for their time.

Procedure and stimuli. During fMRI scans, subjects actively generated or passively listened to short (200 ms) $1 \mathrm{kHz}$ tones. During active experimental trials, subjects were visually cued by the word "PLAY" appearing on a computer screen to actively generate the $1 \mathrm{kHz}$ tones. During the passive experimental trials, subjects were visually cued by the word "LISTEN" and passively listened to a playback of the tones that were generated earlier during the active trials. The active and passive trials alternated, and the auditory stimulus in the passive trials was always a replay of the auditory stimulus generated in the preceding active trials. Thus, the auditory stimulation across each pair of active-passive trials was identical. During silent resting periods, subjects fixated on a cross ("+") presented on the screen. Each run started and ended with a blank screen for $20 \mathrm{~s}$ and contained 32 experimental trials (16 active and 16 passive). Each experimental trial lasted $6 \mathrm{~s}$ and was followed by a silent resting period of $10 \mathrm{~s}$ (Fig. 1).

Tone generation during the active trials was performed in a different manner across the following different experimental runs: "Button $1 \mathrm{~Hz}$ " (during this run, subjects generated the tones by pressing a button with their right index finger at a rate of $1 \mathrm{~Hz}$ ); "Button $2 \mathrm{~Hz}$ " (during this run, subjects generated the tones by pressing a button with their right index finger at a rate of $2 \mathrm{~Hz}$ ); and "Glove $1 \mathrm{~Hz}$ " (during this run, subjects generated the tones by making press-like flexion movements in the air with their right index finger at a rate of $1 \mathrm{~Hz}$ ). Movements in the "Glove $1 \mathrm{~Hz}$ " condition were detected by an MR-compatible motion detection glove (5DT Data Glove 14 MRI, Fifth Dimension Technologies) and used to trigger the tones. During the button runs, tones were triggered by a button press, while during the Glove $1 \mathrm{~Hz}$ run, tones were triggered when index finger flexion crossed a threshold of 0.5 , where 0 corresponds to $0^{\circ}$ of finger position relative to the palm and 1 corresponds with $90^{\circ}$ (full flexion). Subjects wore the glove during all experimental runs.

The rational for using the glove run was to decouple motor output from tactile feedback. Thus, runs in which the tones were generated by button presses involved both a motor component and tactile feedback; conversely, runs in which tones were generated by press-like flexion movements in the air involved the motor component but significantly reduced tactile feedback.

To monitor subjects' attention during the passive conditions, they performed an oddball detection task $(300 \mathrm{~ms}, 0.5 \mathrm{kHz}$ tone; two oddball trials per run, each trial containing two oddball sounds). Detection of such oddballs was reported by a button press, and these passive trials (and their corresponding active trials) were discarded from further analysis, leaving 14 trials per condition. The order of experimental runs was randomized across subjects.

We also assessed the magnitude of activity in auditory regions evoked by button presses alone. Subjects underwent two 4.4 min runs, during which they pressed a button with their right index finger without receiving any auditory feedback. Button presses were visually cued by the word "PRESS," which changed to " $* \star * \star \star *$ following each press. Similar to the button runs, during experimental trials, subjects were required to press the button at a rate of either 1 or $2 \mathrm{~Hz}$ (two separate runs) for periods of $6 \mathrm{~s}$, alternating with resting periods of $10 \mathrm{~s}$. These silent runs preceded all other experimental runs to avoid potential auditory imagery due to association between button presses and auditory feedback from the following experimental runs. The order of these silent control runs $(1$ or $2 \mathrm{~Hz})$ was counterbalanced across subjects. Before each active run, subjects were briefly exposed to tones delivered in the compatible rate $(1$ or $2 \mathrm{~Hz})$ as a reference for performing the finger movements at the desired rate.

Finally, subjects also performed a 6 min auditory localizer run in which they passively listened to short $0.5 \mathrm{kHz}$ tones delivered at a rate of $2 \mathrm{~Hz}$. The localizer run consisted of alternating experimental trials $(6 \mathrm{~s})$ and silent resting periods (10 s). During the localizer run, subjects were engaged in a $1 \mathrm{kHz}$ oddball detection task (two oddball trials, which were discarded from analysis). Data from this run were used for independently defining auditory regions of interest (ROIs) using a contrast of listen $>$ rest.

During the active and passive conditions, auditory stimulation was delivered with MR-compatible OPTOACTIVE headphones (Optoacoustics). All auditory stimuli included a linear rise/decay time of $25 \mathrm{~ms}$.

fMRI data acquisition. Functional imaging was performed on a $3 \mathrm{~T}$ General Electric scanner with an 8-channel head coil located at the Sourasky Medical Center (Tel Aviv, Israel). For each subject, 26 interleaved, ascending, echoplanar $\mathrm{T} 2{ }^{\star}$-weighted slices were acquired, providing whole-brain coverage, not including the cerebellum (slice thickness, $4 \mathrm{~mm}$; slice gaps, $0 \mathrm{~mm}$; in-plane resolution, $1.72 \times 1.72 \times 4$ $\mathrm{mm}$; TR, $2000 \mathrm{~ms}$; TE, $30 \mathrm{~ms}$; flip angle, $90^{\circ}$; field of view, $220 \times 220$ $\mathrm{mm}^{2}$; matrix size, $128 \times 128$ ). For anatomical reference, a whole-brain high-resolution T1-weighted scan (voxel size, $1 \times 1 \times 1 \mathrm{~mm}$ ) was acquired for each subject.

fMRI data preprocessing and analysis. fMRI data analysis was performed using the Brain Voyager QX version 2.3.1 software package (Brain Innovation). Preprocessing of functional data included cubic spline slice time correction, trilinear/sinc 3D motion correction, and temporal high-pass filtering of 2 cycles per run.

The preprocessed functional data were registered to anatomical data and then transformed into the standardized Talairach coordinate system (Talairach and Tournoux, 1988). Data analysis was performed using the general linear model (GLM). Auditory ROIs were defined in superior temporal gyri (STGs; see Fig. 2a) using the auditory localizer data with a GLM contrast of listen $>$ rest whole-brain corrected using false discovery rate (FDR) of $q<0.05$ (Benjamini and Hochberg, 1995). ROIs were defined in individual subjects with a maximum cluster size of $10 \mathrm{~mm}$ in each axis. The percentage of signal change in each trial was calculated relative to the average signal of two time points ( -2 and $0 \mathrm{~s}$ ), with time point 0 corresponding to trial onset. The mean of three time points $(4,6$, and $8 \mathrm{~s}$ relative to trial onset) was taken as the representative response for each trial. Effects of interest were examined using repeated-measures analysis of covariance (ANCOVA) and paired $t$ tests with significance level of $\alpha=0.05$. For visualization purposes only, the multistudy maps displayed in Figures $2 a$ and $4 b$, were generated using spatial smoothing (Gaussian filter, FWHM $=12 \mathrm{~mm}$ ) on functional data of individual subjects.

Functional connectivity analysis. To examine what brain regions are functionally connected with auditory cortex during the active condition, we performed a whole-brain functional connectivity analysis using right and left STG ROIs as seed regions. The regressor for the active condition was generated from the time course of each seed region by replacing the signal during the passive trials with the mean activity during the active trials throughout the experiment. Similarly, the regressor for the passive condition was generated by replacing the signal during the active trials 


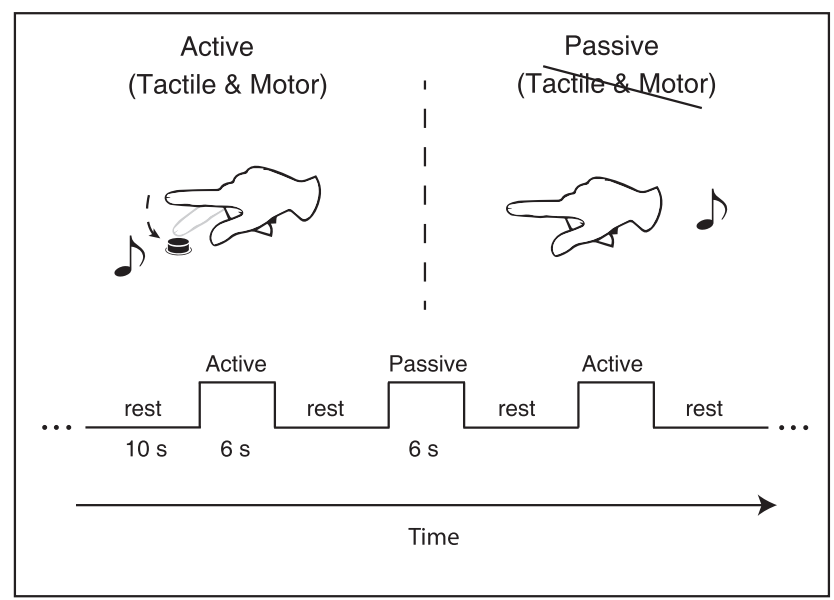

Figure 1. Experimental design. The experiment consisted of alternating trials $(6 \mathrm{~s})$ during which subjects triggered sounds by pressing a button (active condition) or passively listened to the playback of their own sounds recorded during the preceding active trial (passive condition). Experimental trials were separated by $10 \mathrm{~s}$ of silent resting period. The experiment was arranged in two separate runs, during which subjects triggered the sounds at a rate of either 1 or $2 \mathrm{~Hz}$.

with the mean activity during the passive trials throughout the experiment. Finally, all regressors were $z$-score normalized. Such regressors were created based on signals from right and left STGs separately during Button $1 \mathrm{~Hz}$ and Button $2 \mathrm{~Hz}$ conditions. For each STG, we had four main regressors (active/passive in 1 and $2 \mathrm{~Hz}$ execution rates). The design matrix of each subject consisted of regressors from right and left STGs (total of eight experimental regressors). In addition, the design matrix included four confound regressors generated from the corresponding silent execution runs (right/left STGs, and 1 and $2 \mathrm{~Hz}$ conditions). Our functional connectivity contrast of (active right STG + active left STG) > (passive right STG + passive left STG) examined brain regions that are more functionally connected with auditory seed regions during active sound generation compared with passive listening (collapsed across execution rates) above and beyond functional connectivity during silent runs. Multistudy, whole-brain contrast was performed to identify the regions that show the strongest functional connectivity with the seed regions during the active condition compared with the passive condition across subjects.

\section{Results}

Subjects either actively generated or passively listened to $1 \mathrm{kHz}$ pure tones at a rate of either 1 or $2 \mathrm{~Hz}$ (Fig. 1). For each individual subject, we defined auditory ROIs in STG (bilaterally) based on the localizer experiment using a contrast of passive $>$ rest (for details, see Materials and Methods). Figure $2 a$ presents the multisubject map $(n=11)$ using this contrast. ROI selection was restricted to the middle lateral part of STG. Mean \pm standard error of the mean (SEM) Talairach coordinates across subjects: right STG: $x=58.8 \pm 1.4 ; y=-26.6 \pm 2.0 ; z=6.8 \pm 1.5$; left STG: $x=-58.4 \pm 1.3 ; y=-29.6 \pm 1.1 ; z=5.7 \pm 1.2$. These independently defined ROIs were used in subsequent analysis for extracting signal changes across different experimental conditions. During the localizer run, subjects were engaged in an oddball detection task, and correct mean $( \pm$ SEM) detection rate across subjects was $88 \pm 8.72 \%$.

During the active trials of the Button $1 \mathrm{~Hz}$ and Button $2 \mathrm{~Hz}$ runs, subjects pressed a button to trigger the $1 \mathrm{kHz}$ tones using their right hand at the requested rate (mean \pm standard deviation in the Button $1 \mathrm{~Hz}$ condition, $1.06 \pm 0.15 \mathrm{~Hz}$; Button $2 \mathrm{~Hz}$ condition, $2.01 \pm 0.4 \mathrm{~Hz}$ ). The mean correct detection of oddballs in the passive condition across subjects was $100 \%$.
First, we performed a three-way repeated-measures ANOVA examining the effects of condition (active/passive), rate $(1 \mathrm{~Hz} / 2$ $\mathrm{Hz}$ ), and hemisphere (right/left). This analysis revealed a main effect of condition (active/passive: $F_{(1,10)}=15.27, p<0.01$ ) and a main effect of rate $\left(1 \mathrm{~Hz} / 2 \mathrm{~Hz}: F_{(1,10)}=5.16, p<0.05\right)$. Next, we examined the influence of motor output on signal level in auditory cortex. In each subject, we calculated separately the difference in the evoked fMRI signal between each active condition and its corresponding passive conditions (e.g., active vs passive conditions in the Button $1 \mathrm{~Hz}$ condition). We have recently demonstrated that the activity in auditory cortex during the perception of self-generated sounds depends on the identity of the executing effector (right/left hand; Reznik et al., 2014). Therefore, although the ANOVA analysis yielded no significant effect of hemisphere, we examined separately the evoked fMRI signal in right and left STGs. Across subjects, we found that the fMRI signal in left STG was enhanced during both active button conditions ( 1 or $2 \mathrm{~Hz}$ ) compared with their corresponding passive conditions (mean \pm SEM percentage signal change: Button $1 \mathrm{~Hz}: 0.70 \pm 0.11 \%$ active condition; $0.43 \pm 0.10 \%$ passive condition; $n=11, t_{(10)}=2.44$, $p<0.05$, two-tailed paired $t$ test; Button $2 \mathrm{~Hz}: 1.18 \pm 0.21 \%$ active condition; $0.71 \pm 0.12 \%$ passive condition; $t_{(10)}=3.26$, $p<0.01$ ). A similar enhancement pattern was found in right STG, although the difference between active and passive conditions failed to reach statistical significance (Button $1 \mathrm{~Hz}: 0.75 \pm$ $0.12 \%$ active condition; $0.61 \pm 0.13 \%$ passive condition; $t_{(10)}=$ 1.14, $p=0.28$; Button $2 \mathrm{~Hz}: 1.06 \pm 0.17 \%$ active condition; $0.81 \pm 0.14 \%$ passive condition; $t_{(10)}=2.14, p=0.06$ ).

Since the enhanced activity in STGs during active conditions might be explained by mere finger movement regardless of auditory consequences, we also examined the evoked signal in our STG ROIs during silent motor runs (for details, see Materials and Methods). Subjects performed the button presses at the requested rates (Silent $1 \mathrm{~Hz}, 1.08 \pm 0.20 \mathrm{~Hz}$; Silent $2 \mathrm{~Hz}, 2.13 \pm$ $0.23 \mathrm{~Hz}$ ) and were not significantly different from the corresponding rates during sound-producing button conditions (Button $1 \mathrm{~Hz}$ vs Silent $1 \mathrm{~Hz}: n=11, t_{(10)}=0.37, p=0.71$, two-tailed paired $t$ test; Button $2 \mathrm{~Hz}$ vs Silent $2 \mathrm{~Hz}$ : $\left.t_{(10)}=0.87, p=0.40\right)$. In both right and left STG ROIs, the fMRI signal during both 1 and $2 \mathrm{~Hz}$ silent runs was not significantly different than zero (mean \pm SEM percentage signal change for $1 \mathrm{~Hz}$ silent run: right STG: $0.09 \pm 0.13 \% ; n=11, t_{(10)}=0.73, p=0.48$, two-tailed $t$ test; left STG: $0.16 \pm 0.17 \% ; t_{(10)}=0.92, p=0.37 ; 2 \mathrm{~Hz}$ silent run: right STG: $0.06 \pm 0.11 \% ; t_{(10)}=0.56, p=0.58$; left STG: $0.33 \pm 0.18 \%$; $\left.t_{(10)}=1.76, p=0.10\right)$. Moreover, the signal evoked in rightleft STGs during the silent runs was not different across the two rates (right STG: $t_{(10)}=0.32, p=0.75$, left STG: $t_{(10)}=1.34, p=0.2$ ).

Importantly, also after taking into account the activity evoked during the corresponding silent runs ( 1 or $2 \mathrm{~Hz}$ ), signal enhancement during active versus passive conditions in left STG remained significant (repeated-measures ANCOVA, Button $1 \mathrm{~Hz}$ run: $F_{(1,9)}=10.66, p<0.05$; Button $2 \mathrm{~Hz}$ run: $F_{(1,9)}=6.26, p<$ 0.05 ; see Fig. $2 b$ ). Together, the enhanced activity in left STG during the active condition relative to the passive condition cannot be explained by activity evoked by mere finger movements and seems to be better explained by the coupling between a motor action and its corresponding auditory consequence.

To further examine the relationship between the level of activity in motor cortex and fMRI signal modification in auditory cortex, we calculated the correlation between the actual rate in which the sound-producing actions were performed, and the degree of enhanced fMRI signal in right and left STGs. The degree of fMRI signal enhancement in each subject was defined 
as the difference (average percentage signal change) between the active trials and their corresponding passive trials. The execution rate of each subject was calculated by taking the mean rate across all active trials ( 1 or $2 \mathrm{~Hz}$ separately). Using data from both Button $1 \mathrm{~Hz}$ and $2 \mathrm{~Hz}$ conditions from all subjects, and taking into account activity evoked during silent runs, we found that fMRI signal enhancement and actual rate of action execution were significantly correlated in left STG (multiple regression $\beta$ coefficient $=0.39 ; n=$ 22; $p<0.05$, two-tailed $t$ test) but not in right STG $(\beta$ coefficient $=0.11 ; p=0.59$; Fig. $2 c)$. Together, these results suggest that active sound generation with the right hand enhances the fMRI signal in left STG (relative to passive listening to identical sounds); the degree of enhancement covaries with movement rate, and, importantly, this enhancement cannot be explained by signals evoked during silent button pressing.

Next, we used our STG ROIs as seed regions and performed a correlation-based whole-brain functional connectivity analysis (see Materials and Methods). We found two significant patches of voxels showing higher functional connectivity with the seed regions during active sound generation compared with passive listening (Fig. 3). One patch in the middle dorsal part of left superior frontal gyrus (89 voxels; mean Talairach coordinates: $x=$ $-3.6 ; y=-18.2 ; z=52.6$; SMA) and another one in left central sulcus (678 voxels; mean Talairach coordinates: $x=$ $-40.1 ; y=-30.1 ; z=53.6)$. The results support the notion that these regions mediate the signal enhancement in STGs during active sound generation.

During active conditions, subjects pressed a button to generate the auditory stimuli. However, since tactile input has been shown to modulate activity in auditory cortex, this could provide an alternative mechanism explaining our enhanced signal in STGs during active conditions. The importance of this issue is further emphasized by the fact that our functional connectivity analysis points to a patch in the central sulcus (putative M1) as a candidate source of STG modulation. M1 (along the rostral bank and crown of the central sulcus) and primary somatosensory cortex ( $\mathrm{S} 1$; on the caudal bank of the central sulcus and post-central gyrus) are in close proximity. Although we did not apply spatial smoothing to the data, alignment issues (e.g., Talairach coordinates) can still blur the distinction between the two neighboring areas. Therefore, we performed another experiment to examine the role of tactile feedback in STG signal enhancement during active conditions. We used a motion-sensing MR-compatible glove to allow subjects to actively trigger sounds while drastically reducing the amount of tactile feedback in the active sound generation condition (Fig. $4 a$; for details, see Mate-
Left hemisphere

Right hemisphere

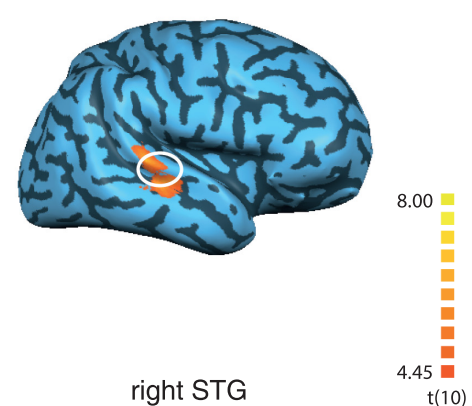

left STG

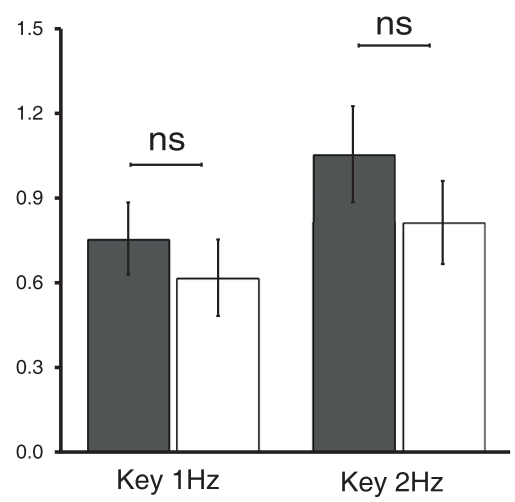

Active

Passive

left STG

right STG

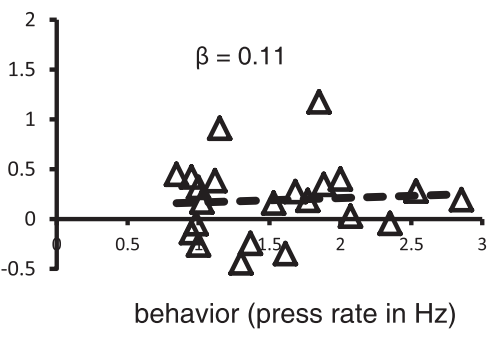

Figure 2. Sensory enhancement in left auditory cortex. $\boldsymbol{a}$, Random effect multistudy map ( $n=11$; FDR corrected using $q<$ 0.05 ) showing a contrast of listen $>$ rest from the auditory localizer run. White circles indicate the auditory ROls in middle superior change) across subjects in Button $1 \mathrm{~Hz}$ and Button $2 \mathrm{~Hz}$ runs was greater in the active compared with passive conditions in left ( $n=$ 11; ANCOVA, ${ }^{*} p<0.05$ ) but not right STG (Button $1 \mathrm{~Hz}, p=0.42$; Button $2 \mathrm{~Hz}, p=0.083$ ). $c$, The enhanced response in left STG covaried with the rate of sound-producing motor output ( $n=11$; multiple regression $\beta$ coefficient, ${ }^{*} p<0.05$ ).

rials and Methods). Subjects performed the sound-producing actions in the Glove $1 \mathrm{~Hz}$ condition at a rate of $0.96 \pm 0.10 \mathrm{~Hz}$, and the mean correct detection of oddballs across subjects in the passive trials was $91 \pm 5.8 \%$.

First, we validated reduced tactile feedback during the active Glove $1 \mathrm{~Hz}$ condition compared with active Button $1 \mathrm{~Hz}$ trials. To this end, we performed a multistudy $(n=11)$ analysis using a contrast of active Button $1 \mathrm{~Hz}>$ active Glove $1 \mathrm{~Hz}$ conditions. We found voxels in left post-central gyrus that show a significantly higher fMRI signal in the active button compared with active Glove $1 \mathrm{~Hz}$ conditions (Fig. 4b). The lower signal in left (contralateral) somatosensory cortex during the active Glove $1 \mathrm{~Hz}$ condition is in agreement with reduced tactile feedback from the right hand.

Next, we examined the enhancement effect (active vs passive) in our STG ROIs. To this end, in an analysis similar to that per- 
Left Right Left Sagittal

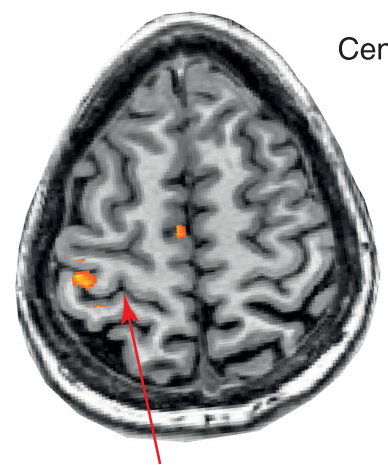

Central Sulcus

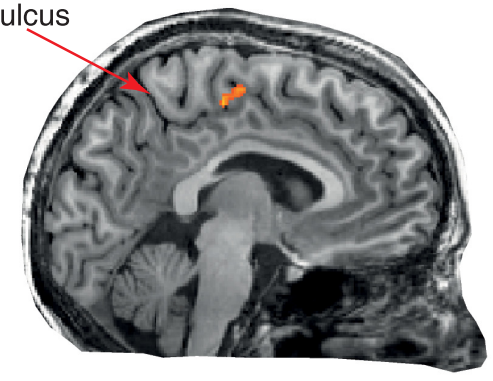

Central Sulcus

Figure 3. Functional connectivity. Random effect multi-study map $(n=11)$ showing the most significant voxels $(p<0.001$ Bonferroni corrected) in active $>$ passive contrast of functional connectivity analysis (see Materials and Methods). Using right and left STGs as a seed region, the activity was most strongly connected with SMA and left M1 during the active compared with the passive condition. a

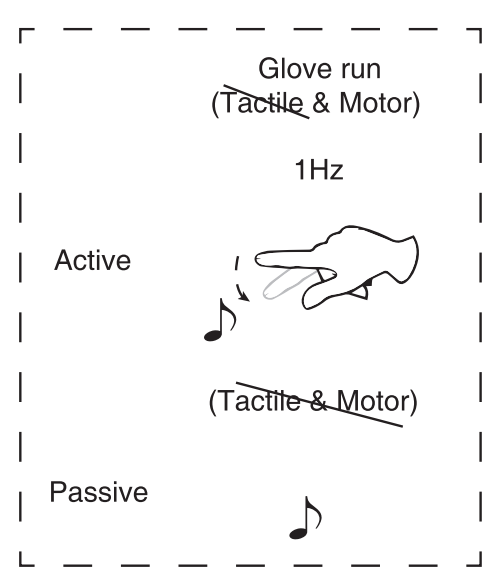

C

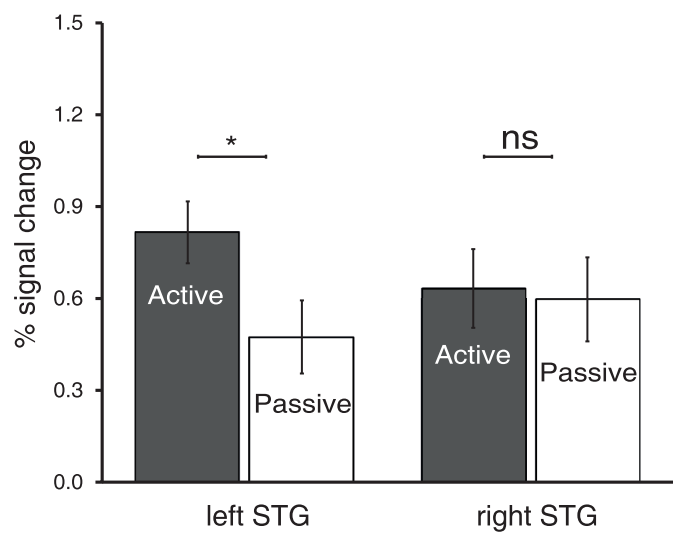

Figure 4. Tactile feedback. $\boldsymbol{a}$, During the active Glove $1 \mathrm{~Hz}$ condition, subjects triggered sounds by flexing a finger in the air without touching a button (see Materials and Methods). $\boldsymbol{b}$, Random-effect multistudy map ( $n=11$; FDR corrected using $q<0.05$ ) showing the contrast of active Button $1 \mathrm{~Hz}>$ active Glove $1 \mathrm{~Hz}$. Significant voxels in left post-central gyrus correspond with decreased somatosensory feedback during active Glove $1 \mathrm{~Hz}$ compared with active Button $1 \mathrm{~Hz}$ conditions $(\mathrm{L} / \mathrm{R}=$ left/right hemisphere, respectively). $c$, Mean \pm SEM response (percentage signal change) across subjects was greater in the active compared with passive Glove $1 \mathrm{~Hz}$ condition in left $S T G\left(n=11\right.$; two-tailed paired $t$ test, $\left.{ }^{*} p<0.05\right)$ but not right STG.d, Mean \pm SEM enhancement effects (active - passive percentage signal change) were not significantly different between Button 1 $\mathrm{Hz}$ and Glove $1 \mathrm{~Hz}$ runs. formed in the button conditions, we compared the evoked fMRI signal (percentage change) in the active and passive conditions during the Glove $1 \mathrm{~Hz}$ runs. Consistent with the Button $1 \mathrm{~Hz}$ run, we found that the fMRI signal across subjects was enhanced during the active compared with passive conditions in left STG $(0.81 \pm 0.10 \%$ active condition; $0.47 \pm$ $0.12 \%$ passive condition; $t_{(10)}=3.43, n=$ $11, p<0.01$, two-tailed paired $t$ test) but not in right STG $(0.63 \pm 0.13 \%$ active condition; $0.59 \pm 0.13 \%$ passive condition; $t_{(10)}=0.38, n=11, p=0.71$; Fig. $4 c$, group average data). We further examined the influence of reduced tactile feedback on the degree of enhancement in left STG by directly comparing the enhancement effect (difference in fMRI signal between active and passive conditions) in the Button $1 \mathrm{~Hz}$ and Glove $1 \mathrm{~Hz}$ runs. We found that the degree of enhancement was not significantly different between the two conditions (mean \pm SEM signal enhancement; Button $1 \mathrm{~Hz}$ run, $0.26 \pm 0.10 \%$; Glove $1 \mathrm{~Hz}$ run, $0.34 \pm 0.10 \% ; n=11$; $t_{(10)}=0.85 ; p=0.41$, two-tailed paired $t$ test; Fig. $4 d$ ). To further examine whether this lack of difference is genuine rather than an issue of statistical power, we applied a Bayesian approach using the Akaike Information criterion (Kass and Raftery, 1995) to examine the validity of the "null hypothesis." We found that the Bayes factor of comparing the modulation effects in auditory ROIs across the two conditions was 0.32 , favoring the claim of a genuine absence of effect (Jeffreys, 1961). Together, these results suggest that the enhanced signal in auditory cortex during active sound generation is less likely to be mediated by tactile feedback and activity in S1.

\section{Discussion}

In the current study, we show that the fMRI signal in left auditory cortex (STG) is stronger during active sound generation compared with passive listening to identical sounds. Furthermore, the degree of this enhancement increases with higher levels of motor output (i.e., faster rate of sound-producing actions). Active sound generation is typically coupled with tactile feedback; therefore, the increased execution rate also corresponds with increased tactile feedback. Since auditory cortex has been shown to respond to passive tactile input, the relative contribution of the two components (motor and tactile) to signal enhancement in STGs is unclear. Using an MR-compatible motion-sensitive glove, we were able to decouple the amount of 
motor output from the amount of tactile feedback and manipulate the two separately. We show that the magnitude of signal enhancement (active minus passive) in left auditory cortex is similar when tactile feedback is dramatically reduced. Together with a functional connectivity analysis, our results support the view that motor output from SMA and contralateral M1 modify activity in auditory cortex during the perception of self-generated sounds.

Our findings are in agreement with previous studies that demonstrate enhanced evoked activity to self-generated stimuli in auditory (Reznik et al., 2014), somatosensory (Simões-Franklin et al., 2011; Ackerley et al., 2012), and visual (Hughes and Waszak, 2011) cortices compared with otherwise identical sensory stimuli perceived in a passive manner. These studies suggest that an efference copy enhances the neural response evoked by the sensory consequences of self-generated actions (see also Christensen et al., 2007). Other studies report attenuated responses to self-generated stimuli and suggest a canceling role of an efference copy in sensory cortices (Blakemore et al., 1998; Martikainen et al., 2005; Aliu et al., 2009; Baess et al., 2009; Saupe et al., 2013; Horváth, 2014; Timm et al., 2014). The conditions in which actions enhance or attenuate evoked activity of their sensory consequences are still not known.

When examining the activity in right and left STGs separately, we found that the fMRI signal in active compared with passive conditions was enhanced in both STGs, although in right STG it failed to reach statistical significance. This finding is in agreement with recent reports (Reznik et al., 2014; Schneider et al., 2014) that activity in auditory cortex during active sound generation is predominantly modified by an efference copy sent from the motor cortex residing in the same hemisphere. In the current study, subjects triggered tones with their right hand, resulting in biased activity to the left motor cortex (contralateral to the executing hand). Since anatomical connections between auditory and motor cortices within the same hemisphere are stronger (Pandya et al., 1969; Romanski and Averbeck, 2009), this might explain the stronger enhancement we observed in left STG compared with right STG.

Further support for the notion that responses in auditory cortex are modulated by efference copies sent from motor cortex, is provided by the demonstration that the magnitude of signal enhancement we found in STG ipsilateral to the active motor cortex (i.e., left STG) is parametrically modulated by the rate of sound-producing actions. This is in agreement with previous studies showing that activity in sensory regions is closely coupled with the magnitude/rate of motor output (Paus et al., 1996; Cui et al., 2014). Importantly, the modulatory role of action execution on activity in auditory cortex was found only when the actions were coupled with sensory feedback (i.e., sounds in our case). When the actions were not coupled with auditory feedback, the signal in auditory cortex was invariant to silent execution rates.

Repeated exposure to identical sound sequences has been shown to result in reduced signal in auditory cortex (i.e., repetition suppression; Bergerbest et al., 2004). Since in our design, the passive condition was an identical replay of the previous active condition, it could be argued that such an effect underlies the lower signal in the passive condition. However, the laterality of the enhancement effect in left STG and its parametric modulation, which covaries with motor output, suggest that a global effect of repetition suppression is unlikely. This is compatible with our previous report (Reznik et al., 2014) in which we found no repetition suppression using short musical sequences.

It has been suggested that the motor system, perhaps through active sensing of the environment, is well suited for sending predictive information regarding upcoming sensory events to the relevant sensory regions (Schubotz, 2007; Schroeder et al., 2010; Arnal and Giraud, 2012). Indeed, a series of studies demonstrated that activity patterns in parietal and frontal cortices can predict action type (grasp/reach) prior to overt action execution (Gallivan et al., $2013 \mathrm{a}, \mathrm{b})$. It has been also suggested that readiness potentials arising before one's intention to perform a voluntary action might carry predictive information regarding upcoming sensory consequences of voluntary actions (Sirigu et al., 2004). Here we postulate that during active sound generation, such predictive information (in the form of an efference copy) is sent from motor to auditory cortices. Within the motor system, our functional connectivity analysis suggests SMA and M1 as candidate regions modifying activity in auditory cortex during active sound generation. The cerebellum has been previously suggested to be involved in modifying activity in secondary somatosensory regions during self-generated tactile stimulation (Blakemore et al., 1998). Additionally, in the auditory domain, patients with lesions in the cerebellum do not exhibit the typical sensory attenuation effect as measured by the N100 ERP component when comparing self-generated to externally generated sounds (Knolle et al., 2012, 2013). Since our whole-brain fMRI scans did not include the cerebellum, we cannot draw any conclusions from the current results regarding its role in modifying activity in auditory cortex.

It should be noted that research in recent years demonstrated that regions in auditory cortex are not exclusively engaged in auditory processing, but are also sensitive to visual (Kayser et al., 2008), tactile (Schroeder et al., 2001; Foxe et al., 2002; Fu et al., 2003; Bolognini et al., 2010), and also olfactory (Cohen et al., 2011) stimulations. These findings are further supported by a recent report (Liang et al., 2013) that even primary sensory regions are responsive to sensory input from other modalities. Nevertheless, our current results suggest that tactile feedback, which is typically coupled with action execution, is not the major source of signal enhancement in auditory cortex response to selfgenerated sounds. This is compatible with a recent EEG study that also manipulated the amount of tactile feedback and compared auditory evoked responses to sounds triggered by actions with or without mechanical impact (button press vs crossing a laser beam; Horváth, 2014). While the EEG results demonstrate that mechanical impact can play a role in signal modulation (sensory suppression), it does not provide a full account of the effect.

Volition and the sense of agency (Haggard, 2008) have been also shown to play a role in the modulation of neural responses to sensory consequences of self-generated actions. For example, the auditory N100 EEG evoked response is reduced (sensory attenuation) for active compared with passive conditions only when sounds in the active condition are triggered voluntarily. When finger movement triggering the sound is induced by transcranial magnetic stimulation of motor cortex, no sensory attenuation is observed (Timm et al., 2014; but for similar findings in proprioception, see Haggard and Whitford, 2004). Thus, the motor act and its accompanying proprioceptive feedback by themselves are not sufficient to modulate activity in auditory cortex, but rather the intention and voluntary aspect of the movement are needed. Another aspect of voluntary actions is predictability-in the active condition, subjects can predict the timing and nature of their action consequences. Indeed, introducing perturbations to ex- 
pected feedback (in space, time, or type of feedback) can abolish sensory modulations at the perceptual or physiological level (Blakemore et al., 1999; Bays et al., 2005; Eliades and Wang, 2008; Desantis et al., 2014).

To conclude, we show that the magnitude of fMRI signal enhancement in STGs to active sound generation compared with passive listening covaries with the magnitude of motor output. Signal enhancement is stronger in auditory cortex residing in the same hemisphere as the active motor cortex (contralateral to the active hand), and tactile feedback does not seem to play a major role in this effect. Our results are in favor of a model in which signals from contralateral M1 and SMA are a driving modulator of auditory cortex during active sound generation. Such signals might carry predictive information regarding the sensory consequences of upcoming actions.

\section{References}

Ackerley R, Hassan E, Curran A, Wessberg J, Olausson H, McGlone F (2012) An fMRI study on cortical responses during active self-touch and passive touch from others. Front Behav Neurosci 6:51. CrossRef Medline

Aliu SO, Houde JF, Nagarajan SS (2009) Motor-induced suppression of the auditory cortex. J Cogn Neurosci 21:791-802. CrossRef Medline

Arnal LH, Giraud AL (2012) Cortical oscillations and sensory predictions. Trends Cogn Sci 16:390-398. CrossRef Medline

Baess P, Widmann A, Roye A, Schröger E, Jacobsen T (2009) Attenuated human auditory middle latency response and evoked $40-\mathrm{Hz}$ response to self-initiated sounds. Eur J Neurosci 29:1514-1521. CrossRef Medline

Bays PM, Wolpert DM, Flanagan JR (2005) Perception of the consequences of self-action is temporally tuned and event driven. Curr Biol 15:11251128. CrossRef Medline

Benjamini Y, Hochberg Y (1995) Controlling the false discovery rate-a practical and powerful approach to multiple testing. J R Stat Soc Series B Stat Methodol 57:289-300.

Bergerbest D, Ghahremani DG, Gabrieli JDE (2004) Neural correlates of auditory repetition priming: reduced fMRI activation in the auditory cortex. J Cogn Neurosci 16:966-977. CrossRef Medline

Blakemore SJ, Wolpert DM, Frith CD (1998) Central cancellation of self-produced tickle sensation. Nat Neurosci 1:635-640. CrossRef Medline

Blakemore SJ, Frith CD, Wolpert DM (1999) Spatio-temporal prediction modulates the perception of self-produced stimuli. J Cogn Neurosci 11: 551-559. CrossRef Medline

Bolognini N, Papagno C, Moroni D, Maravita A (2010) Tactile Temporal Processing in the Auditory Cortex. J Cogn Neurosci 22:1201-1211. CrossRef Medline

Christensen MS, Lundbye-Jensen J, Geertsen SS, Petersen TH, Paulson OB, Nielsen JB (2007) Premotor cortex modulates somatosensory cortex during voluntary movements without proprioceptive feedback. Nat Neurosci 10:417-419. CrossRef Medline

Cohen L, Rothschild G, Mizrahi A (2011) Multisensory integration of natural odors and sounds in the auditory cortex. Neuron 72:357-369. CrossRef Medline

Crapse TB, Sommer MA (2008) Corollary discharge across the animal kingdom. Nat Rev Neurosci 9:587-600. CrossRef Medline

Cui F, Arnstein D, Thomas RM, Maurits NM, Keysers C, Gazzola V (2014) Functional magnetic resonance imaging connectivity analyses reveal efference-copy to primary somatosensory area, BA2. PLoS One 9:e84367. CrossRef Medline

Desantis A, Roussel C, Waszak F (2014) The temporal dynamics of the perceptual consequences of action-effect prediction. Cognition 132:243250. CrossRef Medline

Eliades SJ, Wang X (2003) Sensory-motor interaction in the primate auditory cortex during self-initiated vocalizations. J Neurophysiol 89:21942207. CrossRef Medline

Eliades SJ, Wang X (2008) Neural substrates of vocalization feedback monitoring in primate auditory cortex. Nature 453:1102-1106. CrossRef Medline

Foxe JJ, Wylie GR, Martinez A, Schroeder CE, Javitt DC, Guilfoyle D, Ritter
W, Murray MM (2002) Auditory-somatosensory multisensory processing in auditory association cortex: an fMRI study. J Neurophysiol 88:540 543. Medline

Fu KM, Johnston TA, Shah AS, Arnold L, Smiley J, Hackett TA, Garraghty PE, Schroeder CE (2003) Auditory cortical neurons respond to somatosensory stimulation. J Neurosci 23:7510-7515. Medline

Gallivan JP, McLean DA, Valyear KF, Culham JC (2013a) Decoding the neural mechanisms of human tool use. Elife 2:e00425. CrossRef Medline

Gallivan JP, Chapman CS, McLean DA, Flanagan JR, Culham JC (2013b) Activity patterns in the category-selective occipitotemporal cortex predict upcoming motor actions. Eur J Neurosci 38:2408-2424. CrossRef Medline

Haggard P (2008) Human volition: towards a neuroscience of will. Nat Rev Neurosci 9:934-946. CrossRef Medline

Haggard P, Whitford B (2004) Supplementary motor area provides an efferent signal for sensory suppression. Cogn Brain Res 19:52-58. CrossRef

Horváth J (2014) The role of mechanical impact in action-related auditory attenuation. Cogn Affect Behav Neurosci 14:1392-1406. CrossRef Medline

Hughes G, Waszak F (2011) ERP correlates of action effect prediction and visual sensory attenuation in voluntary action. Neuroimage 56:16321640. CrossRef Medline

Hughes G, Desantis A, Waszak F (2013) Mechanisms of intentional binding and sensory attenuation: the role of temporal prediction, temporal control, identity prediction, and motor prediction. Psychol Bull 139:133-151. CrossRef Medline

Jeannerod M (2003) The mechanism of self-recognition in humans. Behav Brain Res 142:1-15. CrossRef Medline

Jeffreys H (1961) The theory of probability, Ed 3. Oxford, UK: Oxford UP.

Kass RE, Raftery AE (1995) Bayes factors. J Am Stat Assoc 90:773-795. CrossRef

Kayser C, Petkov CI, Logothetis NK (2008) Visual modulation of neurons in auditory cortex. Cereb Cortex 18:1560-1574. CrossRef Medline

Knolle F, Schroger E, Baess P, Kotz SA (2012) The cerebellum generates motor-to-auditory predictions: ERP lesion evidence. J Cogn Neurosci 24:698-706. CrossRef

Knolle F, Schröger E, Kotz SA (2013) Cerebellar contribution to the prediction of self-initiated sounds. Cortex 49:2449-2461. CrossRef Medline

Liang M, Mouraux A, Hu L, Iannetti GD (2013) Primary sensory cortices contain distinguishable spatial patterns of activity for each sense. Nat Commun 4:1979. CrossRef

Martikainen MH, Kaneko K, Hari R (2005) Suppressed responses to selftriggered sounds in the human auditory cortex. Cereb Cortex 15:299302. CrossRef Medline

Pandya DN, Hallett M, Kmukherjee SK (1969) Intra-and interhemispheric connections of the neocortical auditory system in the rhesus monkey. Brain Res 14:49-65. CrossRef Medline

Paus T, Perry DW, Zatorre RJ, Worsley KJ, Evans AC (1996) Modulation of cerebral blood flow in the human auditory cortex during speech: role of motor-to-sensory discharges. Eur J Neurosci 8:2236-2246. CrossRef Medline

Poulet JF, Hedwig B (2007) New insights into corollary discharges mediated by identified neural pathways. Trends Neurosci 30:14-21. CrossRef Medline

Reznik D, Henkin Y, Schadel N, Mukamel R (2014) Lateralized enhancement of auditory cortex activity and increased sensitivity to self-generated sounds. Nat Commun 5:4059. CrossRef Medline

Romanski LM, Averbeck BB (2009) The primate cortical auditory system and neural representation of conspecific vocalizations. Annu Rev Neurosci 32:315-346. CrossRef Medline

Saleem AB, Ayaz A, Jeffery KJ, Harris KD, Carandini M (2013) Integration of visual motion and locomotion in mouse visual cortex. Nat Neurosci 16:1864-1869. CrossRef Medline

Saupe K, Widmann A, Trujillo-Barreto NJ, Schröger E (2013) Sensorial suppression of self-generated sounds and its dependence on attention. Int J Psychophysiol 90:300-310. CrossRef Medline

Schneider DM, Nelson A, Mooney R (2014) A synaptic and circuit basis for corollary discharge in the auditory cortex. Nature 513:189-194. CrossRef Medline

Schroeder CE, Lindsley RW, Specht C, Marcovici A, Smiley JF, Javitt DC 
(2001) Somatosensory input to auditory association cortex in the macaque monkey. J Neurophysiol 85:1322-1327. Medline

Schroeder CE, Wilson DA, Radman T, Scharfman H, Lakatos P (2010) Dynamics of active sensing and perceptual selection. Curr Opin Neurobiol 20:172-176. CrossRef Medline

Schubotz RI (2007) Prediction of external events with our motor system: towards a new framework. Trends Cogn Sci 11:211-218. CrossRef Medline

Schürmann M, Caetano G, Hlushchuk Y, Jousmäki V, Hari R (2006) Touch activates human auditory cortex. Neuroimage 30:1325-1331. CrossRef Medline

Simões-Franklin C, Whitaker TA, Newell FN (2011) Active and passive touch differentially activate somatosensory cortex in texture perception. Hum Brain Mapp 32:1067-1080. CrossRef Medline

Sirigu A, Daprati E, Ciancia S, Giraux P, Nighoghossian N, Posada A, Haggard
P (2004) Altered awareness of voluntary action after damage to the parietal cortex. Nat Neurosci 7:80-84. CrossRef Medline

Sperry RW (1950) Neural basis of the spontaneous optokinetic response produced by visual inversion. J Comp Physiol Psychol 43:482-489. CrossRef Medline

Talairach J, Tournoux P (1988) Co-planar stereotaxic atlas of the human brain. 3-dimensional proportional system: an approach to cerebral imaging. New York: Thieme.

Timm J, SanMiguel I, Keil J, Schröger E, Schönwiesner M (2014) Motor intention determines sensory attenuation of brain responses to selfinitiated sounds. J Cogn Neurosci 26:1481-1489. CrossRef Medline

von Holst E (1954) Relations between the central nervous system and the peripheral organs. Anim Behav 2:89-94. CrossRef

Wolpert DM, Ghahramani Z, Jordan MI (1995) An internal model for sensorimotor integration. Science 269:1880-1882. CrossRef Medline 